\title{
Effects of glucocorticoid on the expression and regulation of aquaporin 5 in the paranasal sinus of rats with chronic rhinosinusitis
}

\author{
CHEN-JIE YU ${ }^{1,2^{*}}$, XIN-YAN CUI ${ }^{3 *}$, LING LU $^{2}, \mathrm{JUN} \mathrm{YANG}^{4}, \mathrm{BIN} \mathrm{CHEN}^{5}, \mathrm{CHENG} \mathrm{WEN} \mathrm{ZHU}^{2}$ and XIA GAO ${ }^{1,2}$ \\ ${ }^{1}$ Department of Otolaryngology, Drum Tower Clinical Medical School, Nanjing Medical University; \\ ${ }^{2}$ Department of Otolaryngology, Nanjing Drum Tower Hospital, Nanjing University School of Medicine, Nanjing, \\ Jiangsu 210008; ${ }^{3}$ Department of Otolaryngology, The First Affiliated Hospital, Nanjing Medical University, Nanjing, \\ Jiangsu 210029; ${ }^{4}$ Department of Pathology, Nanjing Drum Tower Hospital, Nanjing University School of Medicine, \\ Nanjing, Jiangsu 210008; ${ }^{5}$ School of Medicine, Southeast University, Nanjing, Jiangsu 210009, P.R. China
}

Received January 8, 2016; Accepted December 23, 2016

DOI: $10.3892 /$ etm.2017.4215

\begin{abstract}
Aquaporins (AQPs) are water-specific membrane channel proteins that regulate water homeostasis for cells and organisms. AQP5 serves an important role in the maintenance of mucosal water homeostasis, and potentially contributes to mucosal edema and inflammation formation in chronic rhinosinusitis (CRS). The aim of the present study was to explore the expression pattern of AQP5 and the effect of glucocorticoids on AQP5 expression in rats with CRS. The rats were randomly divided into three equal groups, as follows: CRS, dexamethasone (dexa) treatment and control groups. A polyvinyl acetal material containing Staphylococcus aureus was inserted into the left nasal cavity of each rat from the CRS and dexa groups. On the 90th post-operative day, the dexa group received dexamethasone ( $2 \mathrm{mg} / \mathrm{kg} /$ day) via intraperitoneal injection for 7 days. The controls did not receive any treatment. The expression of AQP5 in the sinonasal mucosa was determined using immunohistochemistry and quantitative PCR. The immunoreactivities of AQP5 were primarily noted in the epithelial lining and glandular cells, the vascular endothelium and in the goblet cells in the sinonasal mucosa. The AQP5 mRNA expression level was significantly higher in the dexa group than in the control and CRS groups $(\mathrm{P}=0.006$ and $\mathrm{P}=0.014$, respectively). However, no significant difference was indicated between the CRS and control groups $(\mathrm{P}=0.760)$. In conclusion, the current study suggests that glucocorticoids induce AQP5 expression in
\end{abstract}

Correspondence to: Professor Xia Gao, Department of Otolaryngology, Drum Tower Clinical Medical School, Nanjing Medical University, 321 Zhongshan Road, Gulou, Nanjing, Jiangsu 210008, P.R. China

E-mail: xiagaogaoent@163.com

*Contributed equally

Key words: aquaporin 5, chronic rhinosinusitis, glucocorticoid the sinonasal mucosa of CRS rats, which highlights AQP5 as a potential target in the diagnosis and treatment of CRS.

\section{Introduction}

Chronic rhinosinusitis (CRS) is a group of disorders defined as persistent inflammation involving the nose and paranasal mucosa, and is characterized by nasal blockage/obstruction/congestion, nasal discharge, facial pain/pressure, and/or reduction in or loss of smell (1). The overall prevalence of CRS ranged from 6.9 to $27.1 \%$ (mean, $10.9 \%$ ) due to obvious geographical variation (2), which causes a significant public health problem as well as considerable socioeconomic costs $(1,3)$. Currently, not all CRS cases can be completely controlled due to the complex and multifactorial etiology of this condition, including the involvement of exogenous pathogens, microbial biofilms and individual host factors $(4,5)$.

Glucocorticoids are effectively used as anti-inflammatory drugs to improve airway inflammatory diseases, and are considered as the first-line treatment for CRS $(1,6,7)$. In addition, glucocorticoids have been reported to regulate the water balance in various tissues and organs, including the lungs, peritoneum and middle ear (8).

Aquaporin 5 (AQP5) is a crucial protein formed by four subunits that passively transports water in and out of cells in accordance with the osmotic gradient across the membrane (9). AQP5 has been described to serve a role in several diseases associated with dysfunction of water regulation, and is key in this process (10-12). Furthermore, differences in the cellular location and mRNA expression pattern of AQP5 were observed in the nasal tissues from CRS patients compared with those of healthy controls $(13,14)$.

Based on their hypothesized functions, glucocorticoids may also alleviate edema and improve the symptoms of CRS by means of their regulatory role on AQP5. No previous studies have examined the effects of glucocorticoids on AQP5 in CRS, to the best of our knowledge. In the present study, the expression pattern of AQP5 and the effect of glucocorticoids on AQP5 expression were studied using a rat model of CRS. 

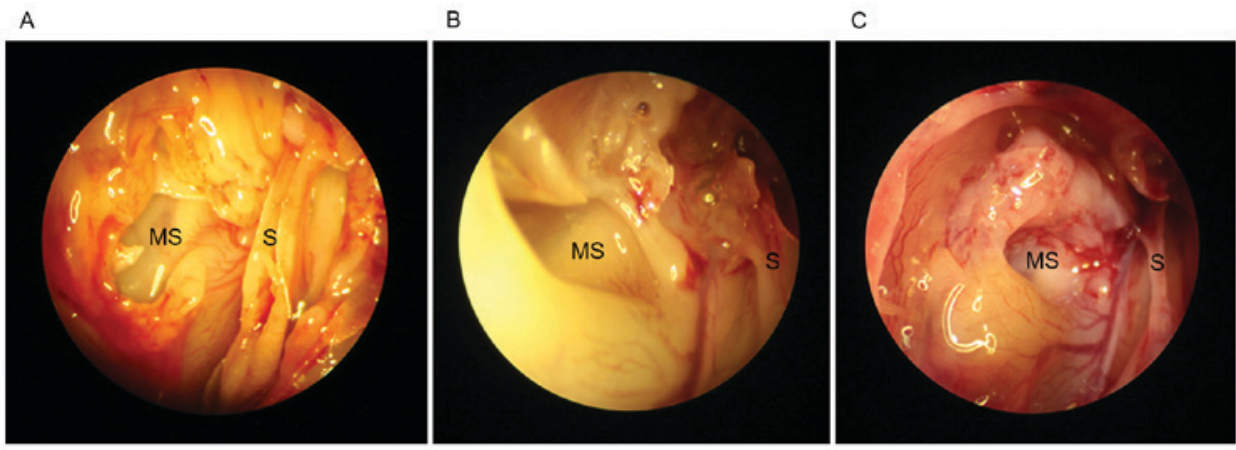

Figure 1. Images of nasoendoscopy in rats of (A) control; (B) CRS; and (C) dexa groups. CRS, chronic rhinosinusitis; dexa, dexamethasone; MS, maxillary sinus; $\mathrm{S}$, septum.

\section{Materials and methods}

Animal model and treatment. A total of 30 male Sprague-Dawley rats (age, 6 months; Animal Laboratory Center, Nanjing Drum Tower Hospital, Nanjing, China) weighing between 220 and $250 \mathrm{~g}$ were used in this study. All animals were handled according to the guidelines of the Animal Care and Use Committee of Nanjing Drum Tower Hospital, Nanjing University School of Medicine.

The rats were randomly divided into three equal groups, as follows: i) CRS; ii) dexamethasone (dexa) treatment; and iii) control groups. Animals in the first two groups were anesthetized by intraperitoneal injection of a mixture of ketamine (50 mg/kg; Jiangsu Hengrui Pharmaceutical Co., Ltd., Jiangsu, China) and diazepam (5 mg/kg; Tianjin Jinyao Pharmaceutical Co.,Ltd., Tianjin, China), and a polyvinyl acetal material (3x5 mm) with Staphylococcus aureus (ATCC 25923; American Type Culture Collection, Manassas, VA, USA), produced in the Laboratory of Clinical Microbiology, Nanjing Drum Tower Hospital) was inserted into the left nasal cavity of each rat from the CRS and dexa groups. The control group did not receive any treatment. On the 90th postoperative day, the dexa group received dexa $(2 \mathrm{mg} / \mathrm{kg} /$ day $)$ via intraperitoneal injection for 7 days. All rats were sacrificed under anesthesia via an intraperitoneal injection of a mixture of ketamine $(50 \mathrm{mg} / \mathrm{kg})$ and diazepam $(5 \mathrm{mg} / \mathrm{kg})$ on the $97 \mathrm{th}$ postoperative day, and the left sinonasal mucosa were removed for subsequent experiments (Fig. 1). Some samples were used immediately for histology and immunohistochemical staining, others were stored in eppendorf tubes at $-70^{\circ} \mathrm{C}$.

Histology and immunohistochemical staining. The samples were prepared by cardiac perfusion with physiological saline, and were then fixed with $4 \%$ paraformaldehyde for $24 \mathrm{~h}$ at $4^{\circ} \mathrm{C}$. The specimens were dehydrated by a graded ethanol series and embedded in paraffin. Tissues were cut into $4-\mu \mathrm{m}$ sections, deparaffinized, and hydrated with phosphate-buffered saline (PBS; pH 7.4). A number of sections were stained with hematoxylin and eosin (HE) for morphological examination, as described previously (15), whereas other samples from the same rats were treated with $3 \% \mathrm{H}_{2} \mathrm{O}_{2}$ for $30 \mathrm{~min}$ at room temperature to block the endogenous peroxide, and then incubated with rabbit polyclonal antibody against AQP5 (ab92320; Abcam, Cambridge, UK; 1:800 dilution) for $18 \mathrm{~h}$ at $4^{\circ} \mathrm{C}$. The sections were then washed with PBS and incubated with a free biotin-conjugated anti-rabbit IgG secondary antibody (PV8000; PowerVision Two-Step Histostaining Reagent; Zhongshan Golden Bridge, Beijing, China; 1:100 dilution) for $30 \mathrm{~min}$ at room temperature. The sections were incubated with $0.05 \%$ 3,3-diaminobenzidine and counterstained with Mayer's hematoxylin. The negative control was incubated with $0.01 \mathrm{M}$ PBS instead of the primary antibody.

RNA extraction, reverse transcription, and quantitative PCR ( $q P C R)$. Total RNA of samples from the left sinonasal muscosa was extracted using TRIzol reagent (Invitrogen; Thermo Fisher Scientific Inc., Waltham, MA, USA), and $1 \mu \mathrm{g}$ of total RNA was reverse-transcribed into cDNA using the Transcriptor First Strand cDNA Synthesis kit (Roche Diagnostics, Indianapolis, IN, USA) according to the manufacturer's instructions. qPCR was conducted using specific primers $(10 \mu \mathrm{mol} / \mathrm{l}$; Invitrogen; Thermo Fisher Scientific, Inc.) and SYBR Premix Ex Taq kit (ABI, USA) with an ABI 7900HT Real-Time PCR System (Applied Biosystems; Thermo Fisher Scientific, Inc.). The concentration of mRNA used in each reaction was $1 \mu \mathrm{g} / \mu \mathrm{l}$. The sequences of the primers were as follows: AQP5 forward, 5'-AGGAGAGGA AGA AGACCATCGA-3', and reverse, 5'-TGCTTCAAACTCTTCGTCTTCCTT-3'; $\beta$-actin forward, 5'-CCCATCTATGAGGGTTACGC-3' and reverse, 5'-TTT AATGTCACGCACGATTTC-3'. The amplification reaction consisted of 40 cycles of denaturation $\left(95^{\circ} ; 20 \mathrm{sec}\right)$, annealing $\left(60^{\circ} ; 60 \mathrm{sec}\right)$ and extension $\left(72^{\circ} ; 60 \mathrm{sec}\right)$. The level of mRNA was assessed using the comparative cycle threshold $(\mathrm{Cq})$ method (16), relative to $\beta$-actin.

Statistical analysis. Statistical analyses were performed using SAS software 9.1.3 (SAS Institute Inc., Cary, NC, USA). Comparisons of relative mRNA expression between groups were analyzed using the unpaired Student's t-test. $\mathrm{P}<0.05$ was considered to indicate a statistically significant difference.

\section{Results}

Histology. HE staining revealed no evidence of inflammatory clusters in the mucosa of the maxillary sinus obtained from control rats (Fig. 2A). However, erosions of epithelial cilia, gland damage, vessel dilation, edema and dispersed lymphocytes in the connective tissue were observed in the sinonasal mucosa in the CRS and dexa groups (Fig. 2B and C), which confirmed chronic sinonasal inflammation. 
A

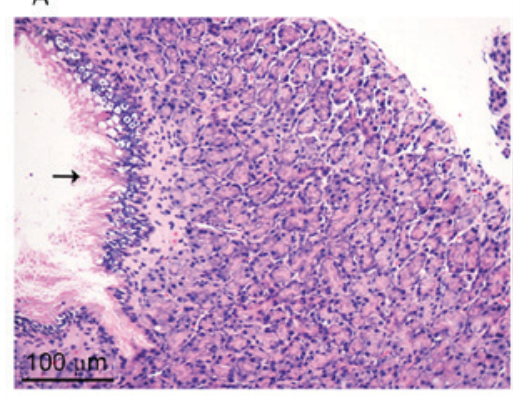

B

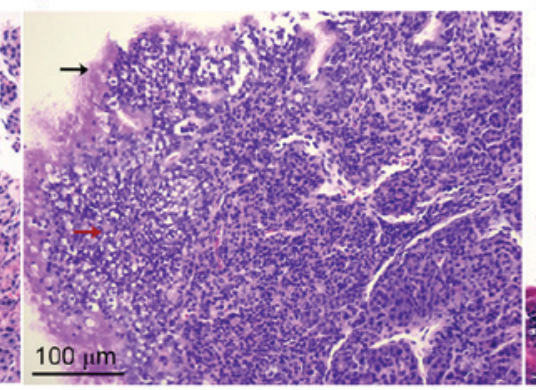

c

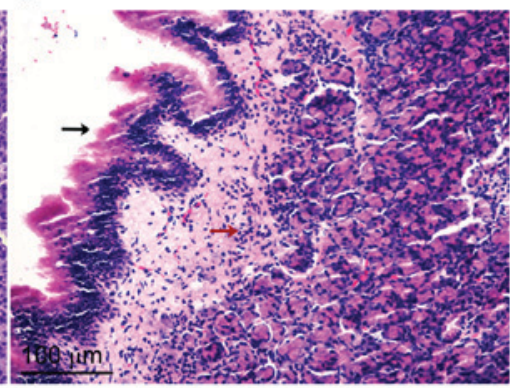

Figure 2. Hematoxylin and eosin staining of the rat sinonasal mucosa from the (A) control; (B) CRS; and (C) dexa groups. Black arrow, ciliated epithelium; red arrow, damaged glands and lymphocyte infiltration; x200 magnification. CRS, chronic rhinosinusitis; dexa, dexamethasone.

A

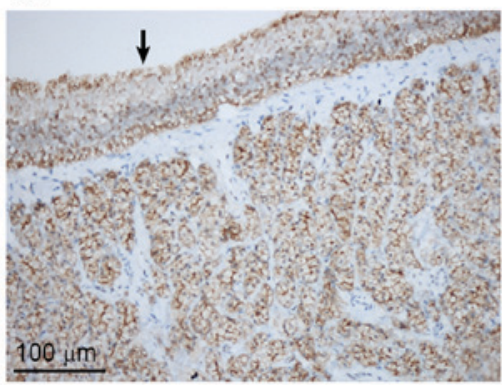

B

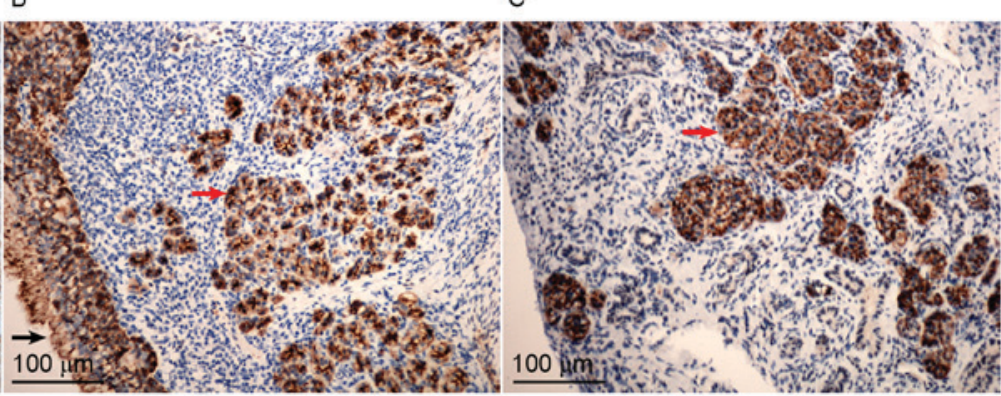

Figure 3. Immunohistochemical localization of aquaporin 5 in the sinonasal mucosa of rats from the (A) control; (B) CRS; and (C) dexa groups. Black arrow, ciliated epithelium; red arrow, glands; brown, positive staining; x200 magnification. CRS, chronic rhinosinusitis; dexa, dexamethasone.

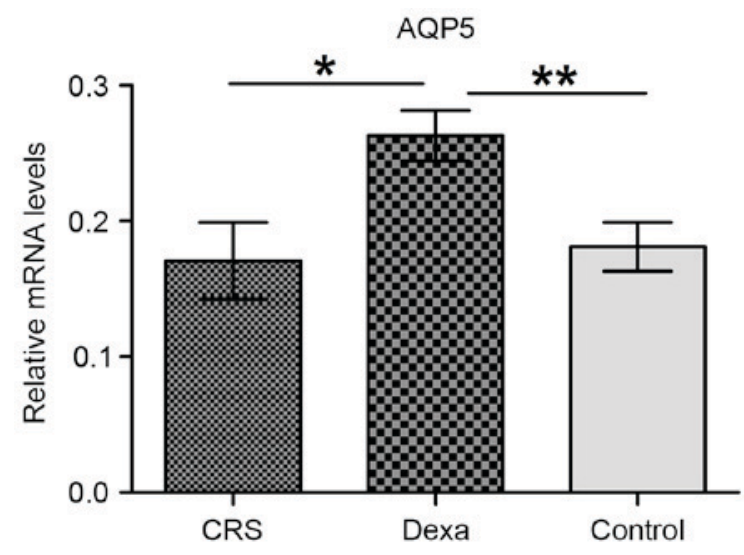

Figure 4. AQP5 mRNA expression levels in the sinonasal mucosa of rats from the CRS, dexa and control groups. Data are presented as the mean \pm standard error of the mean. ${ }^{*} \mathrm{P}<0.05 ;{ }^{* *} \mathrm{P}<0.01$. CRS, chronic rhinosinusitis; dexa, dexamethasone; $\mathrm{AQP} 5$, aquaporin 5.

Immunohistochemical staining. The sinonasal mucosal specimens from all three groups demonstrated positive AQP5 staining, as indicated by the brownish color (Fig. 3). The immunoreactivity of AQP5 was primarily noted in the epithelial lining, glandular cells, vascular endothelium and goblet cells in the sinonasal mucosa (Fig. 3A). No AQP5 expression was observed in the damaged gland areas demonstrating lymphocyte infiltration, whereas a marked reaction was observed in the residual glands (red arrows; Fig. 3B and C).

qPCR. AQP5 mRNA expression was confirmed by qPCR, and was detected in the sinonasal mucosa from all three groups. As reported in Fig. 4, the AQP5 mRNA level was significantly higher in the dexa group than in the CRS and control groups $(\mathrm{P}=0.014$ and $\mathrm{P}=0.006$, respectively). However, no significant difference was found between the CRS and control groups $(\mathrm{P}=0.760)$.

\section{Discussion}

Among the multiple etiological hypotheses of inflammation involving exogenous and host factors, bacterial colonization is the most cited factor $(1,17,18)$. As the most common colonizer of the nasal passages and sinuses, S. aureus is also regarded as a primary causal pathogen of CRS, owing to its high prevalence in CRS patients (17,19), ability to secrete superantigens that alleviate airway inflammation (20) and tendency to form a biofilm, which negatively affects treatment outcomes in CRS patients (21).

In the present study, with the aim of stimulating and maintaining chronic sinonasal inflammation, a model of CRS was established in rats using intranasally administered $S$. aureus. The experimental CRS that developed in the current model is similar to that reported in previous studies $(22,23)$.

AQP5 was confirmed to be primarily expressed in the epithelial lining, subepithelial glandular cells, vascular endothelium and goblet cells of the sinonasal mucosa of the rats. This distribution is consistent with previous findings in humans $(14,24)$. The subepithelial glandular cells are established to participate in mucus secretion and consistency, which maintain the function of the mucociliary system. Furthermore, the epithelial cilia are crucial in osmotic transport to facilitate cilia-dependent movement of mucus. Thus, the present distribution pattern suggests that $\mathrm{AQP} 5$ serves notable and coordinated roles in osmotic homeostasis in the sinonasal mucosa. 
AQP5 mRNA was also detected in the sinonasal mucosa of the rat model. Specifically, the AQP5 mRNA level in the dexa group was significantly higher than that of the other two groups, with no significant difference between the CRS and control groups. It is therefore hypothesized that the underlying reason for this result is as follows: The persistent inflammation induced by S. aureus damaged the natural structure of the nasal mucosa, including the ciliated epithelium and glandular tissue, and AQP5 was predominantly localized to these two cell types. It may be speculated that there may, in fact, be increased expression of AQP5 mRNA in the sinonasal mucosa of CRS rats; however, as the remaining cell counts were reduced in this model, there was an overall similar value of the mRNA quantity compared with the control group.

The valuable role of glucocorticoids in the conservative treatment of CRS is undeniable; in addition to their anti-inflammatory effects, glucocorticoids can regulate the water balance in multiple tissues $(8,25,26)$. Water transport of the sinonasal mucosa is also noteworthy in the pathogenesis of CRS. Abnormal water homeostasis leads to nasal obstruction, purulent discharge and polyp formation. Previous studies revealed that glucocorticoids increased the expression of AQP5 in human airway epithelial cells (25), and may alleviate pulmonary edema in asthmatic rats (26).

In the present study, elevated expression of AQP5 mRNA was observed following glucocorticoid stimulation by intraperitoneal administration for 7 days. It was therefore hypothesized that the regulatory effect of glucocorticoids on AQP5 results in osmotic homeostasis of the nasal mucosa via promotion of glandular secretion and alleviating edema, which consequently improves local inflammation. However, additional studies are required to confirm the protein expression pattern of AQP5, and to elucidate whether glucocorticoids modulate the expression of AQP5 in a dose- and/or time-dependent manner.

In summary, the current study investigated the expression of AQP5 and the effect of glucocorticoids on AQP5 expression in a rat model of CRS. The results demonstrated that glucocorticoids enhance the functional expression of AQP5. These findings may provide evidence for a novel target in CRS treatment.

\section{Acknowledgements}

The current work was supported by the Medical Science Program of Nanjing Municipality (grant no. YKK13081) and the Medical Youth Talent Cultivation Project of Nanjing Municipality (grant no. QRX11193).

\section{References}

1. Fokkens WJ, Lund VJ, Mullol J, Bachert C, Alobid I, Baroody F, Cohen N, Cervin A, Douglas R, Gevaert P, et al: EPOS 2012: European position paper on rhinosinusitis and nasal polyps 2012. A summary for otorhinolaryngologists. Rhinology 50: 1-12, 2012

2. Hastan D, Fokkens WJ, Bachert C, Newson RB, Bislimovska J, Bockelbrink A, Bousquet PJ, Brozek G, Bruno A, Dahlén SE, et al: Chronic rhinosinusitis in Europe-an underestimated disease. A GA²LEN study. Allergy 66: 1216-1223, 2011.

3. Subspecialty Group of Rhinology, Editorial Board of Chinese Journal of Otorhinolaryngology Head and Neck Surgery; Subspecialty Group of Rhinology, Society of Otorhinolaryngology Head and Neck Surgery, Chinese Medical Association: Guidelines for diagnosis and treatment of chronic rhinosinusitis (2012, Kunming). Zhonghua Er Bi Yan Hou Tou Jing Wai Ke Za Zhi 48: 92-94, 2013.
4. Mahoney EJ and Metson R: Palliative care for the patient with refractory chronic rhinosinusitis. Otolaryngol Clin North Am 42: 39-47, vii-viii, 2009.

5. Tan BK, Schleimer RP and Kern RC: Perspectives on the etiology of chronic rhinosinusitis. Curr Opin Otolaryngol Head Neck Surg 18: 21-26, 2010.

6. Brozek JL, Bousquet J, Baena-Cagnani CE, Bonini S, Canonica GW, Casale TB, van Wijk RG, Ohta K, Zuberbier T, Schünemann HJ, et al: Allergic rhinitis and its impact on asthma (ARIA) guidelines: 2010 revision. J Allergy Clin Immunol 126: 466-476, 2010.

7. Okubo K, Kurono Y, Fujieda S, Ogino S, Uchio E, Odajima H and Takenaka H; Japanese Society of Allergology: Japanese guideline for allergic rhinitis 2014. Allergol Int 63: 357-375, 2014.

8. Yu C, Cui X, Chen F, Yang J, Qian X and Gao X: Effect of glucocorticoids on aquaporin-1 in guinea pigs with otitis media with effusion. ExpTher Med 5: 1589-1592, 2013.

9. Arbeithuber B, Thuenauer R, Gravogl Y, Balogi Z, Römer W, Sonnleitner A and Tiemann-Boege I: Aquaporin 5 expression in mouse mammary gland cells is not driven by promoter methylation. Biomed Res Int 2015: 460598, 2015.

10. Wang K, Feng YL, Wen FQ, Chen XR, Ou XM, Xu D, Yang J and Deng ZP: Decreased expression of human aquaporin-5 correlated with mucus overproduction in airways of chronic obstructive pulmonary disease. Acta Pharmacol Sin 28: 1166-1174, 2007.

11. Wang D, Iwata F, Muraguchi M, Ooga K, Ohmoto Y, Takai M, Mori T and Ishikawa Y: Correlation between salivary secretion and salivary AQP5 levels in health and disease. J Med Invest 56 (Suppl): S350-S353, 2009.

12. Yoshimura S, Nakamura H, Horai Y, Nakajima H, Shiraishi H, Hayashi T, Takahashi T and Kawakami A: Abnormal distribution of AQP5 in labial salivary glands is associated with poor saliva secretion in patients with Sjögren's syndrome including neuromyelitis optica complicated patients. Mod Rheumatol 26: 384-390, 2016.

13. Frauenfelder C, Woods C, Hussey D, Ooi E, Klebe S and Carney AS: Aquaporin expression profiles in normal sinonasal mucosa and chronic rhinosinusitis. Int Forum Allergy Rhinol 4: 901-908, 2014.

14. Shikani AH, Sidhaye VK, Basaraba RJ, Shikani HJ, Alqudah MA, Kirk N, Cope E and Leid JG: Mucosal expression of aquaporin 5 and epithelial barrier proteins in chronic rhinosinusitis with and without nasal polyps. Am J Otolaryngol 35: 377-383, 2014.

15. Kmiec Z: J.A. Kiernan. Histological and Histochemical Methods: Theory and Practice. 5th edition, Scion Publishing, 2015, 571 pp. Folia HistochemCytobiol 54: 58-59, 2016.

16. Livak KJ and Schmittgen TD: Analysis of relative gene expression data using real-time quantitative PCR and the 2(-Delta Delta C (T)) Method. Methods 25: 402-408, 2001.

17. Al-Mutairi D and Kilty SJ: Bacterial biofilms and the pathophysiology of chronic rhinosinusitis. Curr Opin Allergy Clin Immunol 11: 18-23, 2011.

18. Vickery TW and Ramakrishnan VR: Bacterial pathogens and the microbiome. Otolaryngol Clin North Am 50: 29-47, 2017.

19. Sachse F, Becker K and Rudack C: Incidence of staphylococcal colonization and of the 753Q Toll-like receptor 2 variant in nasal polyposis. Am J Rhinol Allergy 24: e10-e13, 2010.

20. Pinchuk IV, Beswick EJ and Reyes VE: Staphylococcal enterotoxins. Toxins (Basel) 2: 2177-2197, 2010.

21. Singhal D, Foreman A, Jervis-Bardy J and Wormald PJ: Staphylococcus aureus biofilms: Nemesis of endoscopic sinus surgery. Laryngoscope 121: 1578-1583, 2011.

22. Ahn SK, Jeon SY, Khalmuratov R, Kim DJ, Kim JP, Park JJ and Hur DG: Rat model of staphylococcal enterotoxin B-induced rhinosinusitis. Clin Exp Otorhinolaryngol 1: 24-28, 2008.

23. Liang KL, Jiang RS, Wang J, Shiao JY, Su MC, Hsin CH and Lin JF: Developing a rabbit model of rhinogenic chronic rhinosinusitis. Laryngoscope 118: 1076-1081, 2008.

24. Seno S, Ogawa T, Shibayama M, Kouzaki H and Shimizu T: Expression and localization of aquaporin 1, 2, 3, 4 and 5 in human nasal mucosa. Am J Rhinol Allergy 26: 167-171, 2012.

25. Ben Y, Chen J, Zhu R, Gao L and Bai C: Upregulation of AQP3 and AQP5 induced by dexamethasone and ambroxol in A549 cells. Respir Physiol Neurobiol 161: 111-118, 2008.

26. Dong C, Wang G, Li B, Xiao K, Ma Z, Huang H, Wang X and Bai C: Anti-asthmatic agents alleviate pulmonary edema by upregulating AQP1 and AQP5 expression in the lungs of mice with OVA-induced asthma. Respir Physiol Neurobiol 181: 21-28, 2012. 